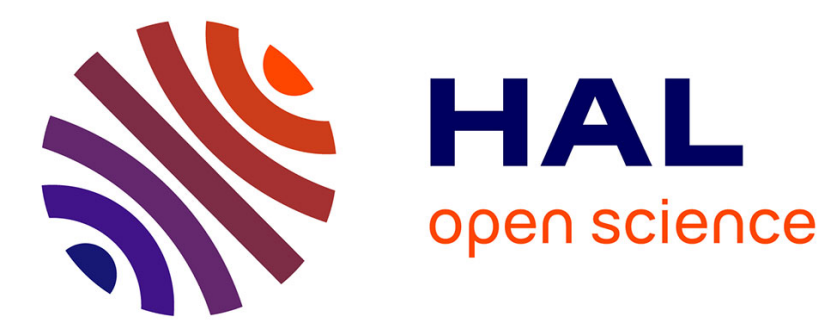

\title{
Multiscale scanning probe microscopy
}

Luc Chassagne, Sylvain Blaize, Pascal Ruaux, Suat Topsu, Pascal Royer, Yasser Alayli, Gilles Lerondel

\section{To cite this version:}

Luc Chassagne, Sylvain Blaize, Pascal Ruaux, Suat Topsu, Pascal Royer, et al.. Multiscale scanning probe microscopy. Review of Scientific Instruments, 2010, 81, pp.086101. 10.1063/1.3473935 . hal00830875

\section{HAL Id: hal-00830875 https://hal.science/hal-00830875}

Submitted on 5 Jun 2013

HAL is a multi-disciplinary open access archive for the deposit and dissemination of scientific research documents, whether they are published or not. The documents may come from teaching and research institutions in France or abroad, or from public or private research centers.
L'archive ouverte pluridisciplinaire HAL, est destinée au dépôt et à la diffusion de documents scientifiques de niveau recherche, publiés ou non, émanant des établissements d'enseignement et de recherche français ou étrangers, des laboratoires publics ou privés. 


\title{
Note: Multiscale scanning probe microscopy
}

\author{
L. Chassagne, ${ }^{1, a}$ S. Blaize ${ }^{2}$ P. Ruaux, ${ }^{1}$ S. Topçu, ${ }^{1}$ P. Royer, ${ }^{2}$ Y. Alayli, ${ }^{1}$ and G. Lérondel ${ }^{2, b)}$ \\ ${ }^{1}$ Laboratoire d'Ingénierie des Systèmes, Université de Versailles Saint-Quentin, 45 Avenue des Etats Unis, \\ 78035 Versailles, France \\ ${ }^{2}$ Laboratoire de Nanotechnologies et d'Instrumentation Optique, Institut Charles Delaunay, \\ UMR CNRS 6279, Université de Technologie de Troyes, 12 rue Marie Curie, BP2060, 10010 Troyes, \\ France
}

(Received 4 May 2010; accepted 10 July 2010; published online 16 August 2010)

Combining the nanoscopic and macroscopic worlds is a serious challenge common to numerous scientific fields, from physics to biology. In this paper, we demonstrate nanometric resolution over a millimeter range by means of atomic-force microscopy using metrological stage. Nanometric repeatability and millimeter range open up the possibility of probing components and materials combining multiscale properties i.e., engineered nanomaterials. Multiscale probing is not limited to atomic-force microscopy and can be extended to any type of scanning probe technique in nanotechnology, including piezoforce microscopy, electrostatic-force microscopy, and scanning near-field optical microscopy. (C) 2010 American Institute of Physics. [doi:10.1063/1.3473935]

Nanotechnology covers numerous fields from physics to biology and multiscale exploration remains a major chalenge. ${ }^{1-5}$ In their behavior, materials and natural artifacts exhibit multiscale properties, presenting sometimes fractal features. ${ }^{6}$ Very Large Scale Integration (VLSI) would not have been possible without instruments able to achieve nanometric resolution over a range of tens of centimeters, namely, the electron beam system combined with an interferometric stage. While scanning probe microscopy can provide atomic resolution and are the most widely used tools, ${ }^{7}$ the investigation range remains limited typically to $100 \mu \mathrm{m}$ but with a resolution of 1024 pixels per line. The investigation range and the resolution are opposite characteristics for traditional systems and are subject to compromise or adaptative techniques. ${ }^{8,11}$ In this era of nanotechnology growth, there is still a need to overcome the limits of the metrological tools currently used to understand the physical features and modeling of the samples. Numerous research laboratories are focusing on overcoming this limitation, using metrological considerations but most of them can achieve nanometric accuracy over a limited range.

Here we report on multiscale scanning probe microscopy (MUSE). This has been achieved thanks to an apparatus combining nanometric repeatability and millimetric range. Multiscale scanning is demonstrated by performing a millimeter long topography image. So far, the main technique to perform long scans is the stitching technique, ${ }^{9,10}$ but it is time-consuming and relies on topographical defects. A first demonstration of a long scan has been already illustrated ${ }^{11}$ but it has a resolution of only $100 \mathrm{~nm}$ and a very long acquisition time, more than $12 \mathrm{~h}$. Because of the high resolution on a macroscopic scale, our system demonstrates that the exploration and the test of components and materials combining multiscale properties and dimensions are pos-

\footnotetext{
a) Author to whom correspondence should be addressed. Electronic mail: luc.chassagne@uvsq.fr.

${ }^{b)}$ Electronic mail: gilles.lerondel@utt.fr.
}

sible. Any macroscopic-scale nanostructured surface, including engineered nanomaterials, can be investigated. Its use is not limited to atomic-force microscopy (AFM) and can be extended to any type of scanning probe technique, including piezoforce microscopy, electrostatic-force microscopy, and scanning near-field optical microscopy.

MUSE is based on a double-stage actuator coupled with an atomic-force microscope. The sketch is illustrated in Fig. 1. The double-stage actuator is composed of commercial linear motors for macroscopic scanning and piezoelectric actuators for fine positioning and error correction. The piezoelectric actuator is a XY model with high-frequency response. Its actuating range is about $3 \mu \mathrm{m}$ for low level voltage control. This range is enough to compensate defects of straightness of the linear motors over $5 \mathrm{~mm}$ with a nanometer repeatability. ${ }^{12}$ A heterodyne interferometer and a specific electronics using phase control principle ensure repeatability and long-term position stability at the nanometric scale ${ }^{13}$ despite nonlinearities of the piezoelectric actuator. The MUSE system also delivers high-rate synchronous pulses with accurate timing to trigger data acquisition for the AFM signal. A specific data acquisition board is used to record all the samples. The AFM model is a commercial one, mechanically adapted to introduce the system beneath the tip. A smooth procedure to approach the cantilever has been used. The XY piezoactuators of the microscope are inhibited since it is replaced by the MUSE interferometric stage. Only the $\mathrm{Z}$ actuator is used to ensure the force feedback. First long scan have been recorded ${ }^{14}$ but thermal constraints have limited the width of the scan to few hundred of nanometers. For this experiment, the system is placed on an antivibration table and in a room where humidity and temperature was controlled. Using MUSE, we have recently demonstrated scanning near-field optical microscopy. ${ }^{15}$ We realized optical image over millimeter range. An integrated optical component was used as a representative example of multiscale components requiring large-scale highly resolved optical mapping. We obtain an 


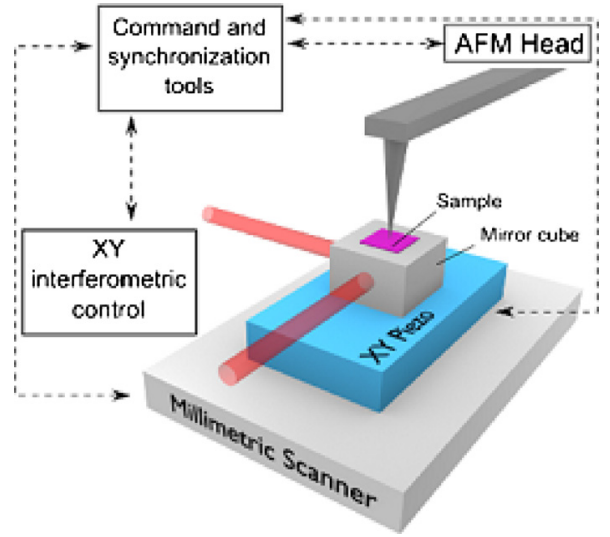

FIG. 1. (Color online) MUSE. Schematic diagram of the double mechanical stage used for scanning under the AFM tip. The stage includes linear motors for long-range scale, a piezoelectric actuator for fine positioning, interferometry for measurements, and high-frequency electronics for synchronization.

image of stationary waves on a millimeter long range. The Fourier analysis of the data was used to calculate the effective index and an uncertainty of a few $10^{-4}$ on the waveguide mode effective index was achieved. While improving the optogeometrical parameter retrieval precision, millimeter scans make the technique sensitive to properties such as optical birefringence. It demonstrates the full interest of multiscale scans while introducing near-field optical metrology.

In this study, a millimeter range topographical image has been realized. The sample used is a silicon on insulator (SOI) optical chip with 1-mm-long submicronic wide waveguiding structures engraved. The structure was fabricated using CMOS photonics technology. The SOI substrate $(200 \mathrm{~mm}$ wafer manufactured by Soitec) used in this study was composed of a monocrystalline silicon layer with a typical thickness of $200 \mathrm{~nm}$ on top of a 1-mm-thick buried oxide layer on a silicon substrate. After a silica hard-mask deposition, the structure pattern was defined by means of a 193-nm-deep UV lithography followed by hard-mask etching and photoresist stripping. The pattern was transferred to the silicon layer by means of a $\mathrm{HBr}$ dry-etching process. The entire waveguiding structure was then covered by a 700-nm-thick cap layer of silica deposited by plasma-enhanced chemical vapor deposition. The expected structure topography is about $200 \mathrm{~nm}$.

The entire waveguiding structure, including the entrance taper and the serpentine area, is clearly revealed by the long range AFM scan on Fig. 2. The parts $a$ and $b$, respectively, show a 1-mm-long AFM image of the optical waveguiding structure and its three-dimensional (3D) representation. The curved area was deliberately designed to probe bend optical losses. The scan range and pixel step are, respectively, of 1.1 $\mathrm{mm}$ long and $20 \mathrm{~nm}$ in the fast-scan direction and $20 \mu \mathrm{m}$ and $100 \mathrm{~nm}$ in the slow-scan direction. We have deliberately limited the longitudinal resolution in order to limit the data file size to make the data analysis easier. It is here worth noting that nanometric resolution can be easily achieved using data acquisition board with higher sample frequency. The scan speed is $40 \mu \mathrm{m} / \mathrm{s}$. The limitation is due to the AFM scan and not to the apparatus that can move as fast as millimeters per second. The part $c$ is a zoom of the purple area corresponding to a standard size AFM scan. The part $d$ is a (a)

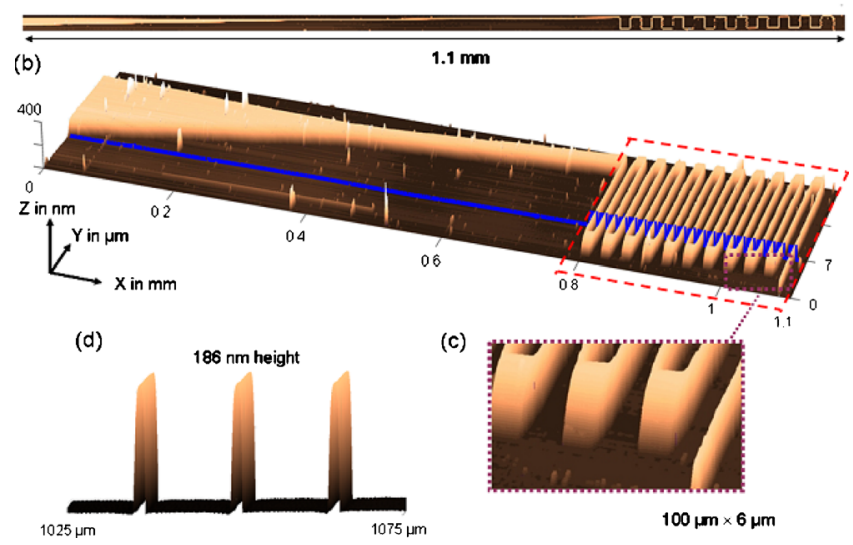

FIG. 2. (Color online) Millimeter-range atomic-force microscopy image $(1.1 \mathrm{~mm} \times 20 \mu \mathrm{m})$. (a) $2 \mathrm{D}$ view of the topography of a SOI sample with embedded waveguiding structures. The resolution of $20 \mathrm{~nm}$ along the $\mathrm{X}$ axis is given by the number of points $-55,000$ for each line; the scan speed is $40 \mu \mathrm{m} / \mathrm{s}$. (b) 3D representation of the image. (c) Zoom of a small area corresponding to a standard size AFM scan. (d) 3D zoom that shows a portion of three waveguides in the serpentine area with high resolution.

second zoom that shows a portion of three waveguides with high resolution in the serpentine area to illustrate nanoscopic zooming capabilities of the acquisition data system.

The large number of points available in the former image is specifically interesting for statistical analysis. Figure 3 shows a typical profile taken along the fast axis as evidenced by the blue line in the part $b$ of Fig. 2. Large-scale oscillations on the left side are due to the waviness of the sample and flatness defects of the mechanical system. Small-scale oscillations on the right side are due to the engraved waveguides. The height of the waveguide has been measured all over the serpentine area as evidenced by the red dotted lines in Fig. 2(b). The dispersion is plotted in the insert of Fig. 3. We find $186.6 \pm 1.4 \mathrm{~nm}(1 \sigma)$ on a statistical set of more than 2000 measurement points. Nanometer scale dispersion is observed showing both the quality of the SOI process relying on CMOS microelectronics standards and the precision of the measurement technique which has been here tremendously improved thanks to the large set of data.

In conclusion, using state of the art SOI photonic component as an example, high resolution atomic-force mapping has been demonstrated over a millimeter range. Besides multiscale imaging, statistical data analysis reveals nanometer

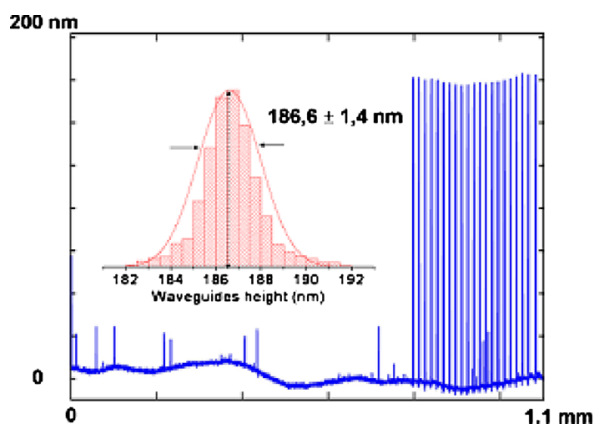

FIG. 3. (Color online) Measurements of the height of the waveguide. The bold line is the cross-sectional view of the bold line in the Fig. 2. The inset curve is the dispersion of the height of the waveguide, calculated over more than 2000 profiles corresponding to the dotted area shown in the Fig. 2. 
precision made here possible thanks to the large set of data available. Such large data acquisition is only possible using highly resolving long range scanning. More generally MUSE opens the door to multiscale metrology that will be a serious challenge in the next future. In addition, a lot of phenomena in natural system present multiscale behavior and at last linking nanoscopic and macroscopic observations can greatly improve the understanding of materials properties.

We thank R. Orobtchouk and J. M. Fedeli from the Institut des Nanosciences de Lyon et CEA/LETI Grenoble for providing us with the SOI sample, and F. Mourgues for mechanical technical support. This research was supported by the French Research Ministry ("nano2m3" integrated project), the Champagne-Ardenne regional authorities, the Ile-de-France regional authorities, and the European Regional Development Fund.
${ }^{1}$ J. Brugger, Nanotechnology 20, 430206 (2009).

${ }^{2}$ A. Engel, Nanotechnology 20, 430202 (2009).

${ }^{3}$ M. Reed, Nanotechnology 20, 430201 (2009).

${ }^{4}$ A. Forchel, Nanotechnology 20, 430204 (2009).

${ }^{5}$ M. Meyyappan, Nanotechnology 20, 430203 (2009).

${ }^{6}$ O. Ovchinnikov, S. Jesse, and S. V. Kalinin, Nanotechnology 20, 255701 (2009)

${ }^{7}$ M. Miles, Nanotechnology 20, 430208 (2009).

${ }^{8}$ C. Recknagel and H. Rothe, Meas. Sci. Technol. 20, 084026 (2009).

${ }^{9}$ R. Bachelot, G. Lérondel, S. Blaize, S. Aubert, A. Bruyant, and P. Royer, Microsc. Res. Tech. 64, 441 (2004).

${ }^{10}$ J. A. Kramar, Meas. Sci. Technol. 16, 2121 (2005).

${ }^{11}$ G. Wilkening and L. Koenders, Nanoscale Calibration Standards and Methods (Wiley-VCH, New York, 2005).

${ }^{12}$ L. Chassagne, M. Wakim, S. Xu, S. Topçu, P. Ruaux, P. Juncar, and Y. Alayli, Meas. Sci. Technol. 18, 3267 (2007).

${ }^{13}$ L. Chassagne, S. Topcu, Y. Alayli, and P. Juncar, Meas. Sci. Technol. 16, 1771 (2005).

${ }^{14}$ A. Sinno, P. Ruaux, L. Chassagne, S. Topçu, Y. Alayli, G. Lérondel, S. Blaize, A. Bruyant, and P. Royer, Rev. Sci. Instrum. 78, 095107 (2007).

${ }^{15}$ G. Lérondel, A. Sinno, L. Chassagne, S. Blaize, P. Ruaux, A. Bruyant, S. Topçu, P. Royer, and Y. Alayli, J. Appl. Phys. 106, 044913 (2009). 